\title{
Linx
}

Revue des linguistes de l'université Paris X Nanterre

49 | 2003

L'actualité des notions d'interlangue et d'interaction exolingue

\section{Interaction exolingue et processus de résolution des malentendus}

\section{Patchareerat Yanaprasart}

\section{(2) OpenEdition \\ Journals}

Édition électronique

URL : http://journals.openedition.org/linx/548

DOI : $10.4000 / \operatorname{lin} x .548$

ISSN : 2118-9692

Éditeur

Presses universitaires de Paris Nanterre

Édition imprimée

Date de publication : 1 décembre 2003

Pagination : 77-90

ISSN : 0246-8743

Référence électronique

Patchareerat Yanaprasart, «Interaction exolingue et processus de résolution des malentendus », Linx [En ligne], 49 | 2003, mis en ligne le 11 mars 2011, consulté le 03 mai 2019. URL : http:// journals.openedition.org/linx/548; DOI : 10.4000/linx.548 


\title{
Interaction exolingue et processus de résolution des malentendus
}

\author{
Patchareerat YANAPRASART \\ CERLE, Université de Fribourg - CLA, Université de Neuchâtel
}

\section{Introduction}

Diverses recherches interactionnistes et sociologiques (Bakhtine 1929/1977, Goffman 1959/19731', Kerbrat-Orecchioni 1990, Bange 1992) envisagent l'échange verbal non pas comme la simple transmission d'un message du destinateur au destinataire, mais comme l'accumulation successive de ce processus dans une situation donnée. De ce point de vue, l'échange verbal n'est plus la communication telle que la définissent les théories sémiotico-linguistiques (Sperber et Wilson 1986/1989), mais l'interaction qui peut être caractérisée à la fois par l'enchainement d'influences mutuelles entre les sujets parlants à travers l'échange verbal et par l'enchaînement de relations constructives entre l'énonciation et la situation environnante. En d'autres termes, il existe une interrelation entre ces trois éléments qui permet au modèle de communication de s'incarner, de s'accumuler et de se donner un cadre concret dans la situation où cet échange s'actualise.

Par conséquent, il n'existe pas d'interaction tout à fait symétrique. Toute interaction est plus ou moins asymétrique (Watzlawick, Helmick-Beavin et Jackson 1967 / 1972, Kerbrat-Orecchioni 1990, Vion 1992), du fait, tout d'abord, des vécus individuels différents, mais aussi de la divergence entre interactants par rapport à la fois à leur «statut» et à leur « rôle » (Linton 1945 / 1967, Flahault 1978, Vion 1992). Outre ces ordres d'asymétrie dits interactionnels, on peut en faire remarquer un autre, si l'on envisage l'interaction exolingue. Il s'agit d'une asymétrie définie en fonction de la "compétence de communication», savoirs et savoir-faire permettant de mener à bien l'interaction.

S'appuyant sur un corpus recueilli entre 1997 et 1999 (Yanaprasart 2000) auprès de Thailandais travaillant avec des Français dans des entreprises françaises

\footnotetext{
${ }^{1}$ Les deux dates renvoient respectivement la première à la première édition, la seconde à l'édition de la traduction française.
} 
implantées en Thailande ainsi qu'auprès d'étudiants thaïs venant apprendre le français en Suisse, cet article a pour but d'observer les stratégies interactionnelles utilisées par les interactants en cas d'asymétrie exolingue et comment ils collaborent pour mener à bien l'interaction, compte tenu de cette asymétrie. Il s'agit de divers contacts exolingues dans la vie professionnelle comme dans la vie quotidienne (une secrétaire thaie et son collègue belge, une standardiste thaïe et un gardien français, une enseignante thaïe et son élève suisse, une étudiante thaïe et un camarade péruvien). Le point commun de ces quatre interactions tient au fait que le français y constitue la langue de communication aussi bien entre natif et non natif qu'entre les non natifs.

\section{Caractéristiques interactionnelles et sociologiques des situations de contact}

Cette description des situations d'interaction asymétrique nous amène à la notion de " communication exolingue », que Porquier (1984) définit comme « celle qui s'établit par le langage par des moyens autres qu'une langue maternelle éventuellement commune aux participants» $(1984: 18)$ et qu'il oppose à d'autres types de communication par des paramètres linguistiques (les compétences linguistiques dont disposent respectivement les interlocuteurs) ou situationnels (comme le milieu linguistique dans lequel se déroule la communication) (ibid. : 29).

Dans la communication, les interactants mettent en œuvre l'ensemble de leurs connaissances, c'est-à-dire les savoirs linguistiques, les savoir-faire et les savoirs socioculturels. On peut alors supposer que les divergences entre les sujets dans une situation d'interaction exolingue apparaissent non seulement au niveau de leur compétence linguistique mais également au niveau d'autres compétences nécessaires pour mener à bien l'interaction, soit par rapport à ce que Hymes (1973/1991 et 1982/1991) appelle « compétence de communication ».

On peut relever dans nos corpus les traces linguistiques qui mettent en relief les divergences entre natif et non natif, et qui permettent de ce fait de situer toutes les situations d'interaction près du "pôle exolingue » sur l'axe proposé par Alber et Py (1985 et 1986). Nous illustrons ci-dessous les divergences de savoirs linguistique et de savoirs socioculturels, sources de malentendus en interaction exolingue.

\section{Les sources de malentendu en interaction exolingue ${ }^{2}$}

N'étant pas à l'aise dans la langue du natif et ayant des difficultés dans la production dans cette langue, le non natif parfois ne peut formuler un énoncé conforme à la norme linguistique, ce qui peut entraîner un malentendu avec le locuteur natif.

\footnotetext{
${ }^{2}$ Lorsque Porquier (1984) parle de la communication exolingue, il ne souligne pas l'aspect interactionnel du contact entre natif et non natif. Pour mettre en relief les caractéristiques interactionnistes et sociologiques que nous avons dégagées dans nos situations, nous parlerons dans le présent travail d'interaction exolingue plutôt que de «communication exolingue ».
} 
D'une façon générale, le malentendu a pour origine le double codage d'une même réalité, c'est-à-dire les interprétations/compréhensions contradictoires d'un même énoncé entre interlocuteurs (Trévise et De Hérédia 1984, De Hérédia 1986 et 1987, De Hérédia-Deprez 1990)

Par ailleurs, la langue qu'utilise le sujet non natif n'est pas la même que celle du natif, mais plutôt une «interlangue» (ou «interlanguage») (Selinker 1972: 214), censée se développer pour atteindre le niveau de la compétence du natif. La production discursive du locuteur non natif est ainsi imparfaite, par exemple, sur les plans syntaxique, sémantique ou phonétique.

\section{Les divergences linguistiques}

\section{Malentendu d'origine phonétique}

Il s'agit du signifiant produit par un locuteur, et qui, n'étant pas reconnu par son interlocuteur, est pris pour un autre, par exemple à cause d'une ressemblance phonétique entre eux (De Hérédia 1986 : 52 et 1987: 24-25, De Hérédia-Deprez 1990 : 217). Ce type de malentendu n'est pas toujours dû à une erreur de réception acoustique chez l'interlocuteur. Il peut tenir également à d'autres facteurs provenant du locuteur, comme une mauvaise prononciation ou un lapsus.

L'observation du premier cas étudié (Conversation 1 ci-dessous) montre que l'un des facteurs importants de malentendu est la prononciation ambiguë ou la mauvaise prononciation par le sujet non natif. Par insuffisance de maitrise de la langue de communication, celui-ci ne peut prononcer correctement un mot ou un segment linguistique. Contre ce facteur d'incompréhension, le natif doit mettre en œuvre des stratégies préventives en concentrant son attention sur le code linguistique. Cette stratégie est observée dans la séquence suivante.

Conversation $1:$ la conversation se déroule entre une Thailandaise $(T)^{3}$ et un Suisse (S) dans un cours de langue ${ }^{4}$; le sujet de discussion est la nourriture contaminée.

\footnotetext{
${ }^{3}$ Même si le profil de chaque personne est fourni en vue d'une meilleure compréhension des textes, toute mention de nom de personne dans les transcriptions renvoie à un pseudonyme choisi pour garantir l'anonymat des participants. Les locuteurs sont donc identifiés par l'initiale de la nationalité. Les données enregistrées par les interactants thaïs ont été transcrites avec les conventions usuelles par nos soins.

Conventions de transcription

..... pause petite/moyenne/plus longue

$/ \backslash \quad$ intonation montante/descendante

extra segment accentué

((rire)) phénomènes non transcrits

: allongement vocalique

[ ] transcription phonétique

$=\quad$ enchainement rapide

(h) aspiration

${ }^{4}$ Cette conversation a été enregistrée à Neuchâtel en 1999 avant un cours de thaï d'une heure hebdomadaire. L'apprenant suisse d'origine espagnole a commencé à apprendre le thaï avec une
} 
1. T on va commencer ..bon=de quoi tu vas me parler..aujourd'hui\

2. S la . la suite de la semaine passée...le poulet Dioxine

3. $\mathrm{T}$ ah oui $\backslash$ c'est un sujet courant . tu manges de la viande/

4. S oui : : mais euh..mais maintenant je ne sais plus quoi manger. la vache folle

ou le poulet de Belgique

5. T mange [plydlegym]

6.S ah/ pourquoi pas de légumes/

7. $\mathrm{T}$ no: : $\mathrm{n}=$ encore [plysdəlegym] $=$ moins de viande

8. S ah j'ai entendu [plydlegym] oui oui (h) j'en mange beaucoup.

9. T bien.. des fruits aussi /

Pour suggérer à S de consommer "davantage" de légumes à cause du problème de la contamination, T utilise le mot plus « mange [plydlegym]» (5T).

On touche là à une difficulté classique du français pour des allophones. D’une part, plus est un «mot servant de comparatif à beaucoup et entrant dans la formation des comparatifs de supériorité et dans celle du superlatif relatif de supériorité » (Le Robert 1993) et peut se prononcer de trois façons: [ply] devant consonne, [plyz] devant voyelle ou [plys] à la finale, ou employé seul. Dans le sens 'désormais... ne pas', c'est-à-dire la négation ne... plus, il se prononce toujours [ply], ou [plyz], facultativement, devant voyelle ("je ne peux plus attendre") 5 .

En voulant signifier 'davantage', la locutrice alloglotte dit d'abord «mange [plydlegym]» (5T), ce qui provoque un malentendu au niveau du signifié : l'allocutaire a compris [plydlegym] comme une négation et se demande pourquoi son enseignante lui déconseille de manger des légumes, et le lui demande: "pourquoi pas de légumes?»(6S). Cette fois, la non-native, s'étant rendu compte du malentendu, procède, pour lever l'ambiguité, à une reformulation, qui recourt à trois moyens conjoints : la prononciation [plys], l'ajout de encore et une précision supplémentaire « plus de viande » (7T), « des fruits aussi » (9T).

Certes, l'alloglotte procède là comme pourrait procéder en pareil cas un locuteur natif, y compris face à un non natif. Mais cet exemple illustre aussi le traitement du malentendu de la part du natif, qui d'abord exprime sa surprise (6S), qu'il ne semble pas attribuer à des raisons linguistiques, puis manifeste ensuite sa compréhension: compréhension du message et élucidation du malentendu, qu'il exprime par des moyens métalinguistiques (8S : «Ah j'ai entendu [ply]...»), faisant écho à la rectification antérieure de $\mathrm{T}$. Il verbalise le malentendu et tente de le résoudre par une question. Cette signalisation directe du problème, au cours de l'interaction, sollicite une explication. Le locuteur natif n’hésite pas à agir de façon explicite sur la situation, tout d'abord parce qu'il sent que la conversation est perturbée. Ensuite, le Suisse s'autorise à prendre l'initiative et à contrôler la co-

étudiante thaillandaise en 1997. Le locuteur suisse a été informé que chaque discussion serait enregistrée et qu'un passage de données serait sélectionné, transcrit et analysé.

${ }^{5}$ Ladite difficulté et l'ambiguiité présente dans le cas évoqué tiennent conjointement à l'absence fréquente de ne en langue parlée et à la variation morphophonologique de plus selon les cas mentionnés. Cette ambiguité pourrait aussi bien se produire entre locuteurs natifs, et être levée comme le fait $\mathrm{T}$ dans $7 \mathrm{~T}$. 
construction du sens avec sa partenaire en raison de son statut et de sa position dominante, due à l'asymétrie des rôles sociaux, des sexes et des âges: même si le Suisse assume un rôle d'apprenant dans cette situation, il est un locuteur natif de la langue d'enseignement, d'apprentissage et de communication. Si son objectif est d'apprendre la langue thaie (matière d'apprentissage), la Thailandaise, elle aussi, est en train d'apprendre le français lorsqu'elle donne des cours dans la langue de l'autre (moyen d'apprentissage). Etant étudiante en français dans la vie quotidienne, elle se réjouit de cette correction considérée par l'un comme par l'autre comme une sousleçon de français. La position linguistiquement dominante du locuteur suisse est rendue encore plus évidente par son rôle socioprofessionnel en tant que cadre d'entreprise, sans parler du respect de la jeune étudiante pour son locuteur en raison de son âge et de l'attitude d'une femme envers un homme selon les règles sociales thaïes. Pour ces raisons, la dimension sociale joue donc un rôle non négligeable dans les comportements discursifs des deux participants. Elle contribue ici au réglage de la communication.

\section{Malentendu d'origine sémantique}

C'est l'attribution erronée d'une valeur à une marque donnée qui provoque ce type de malentendu, lié au fait qu'un même mot recouvre des réalités différentes, qui renvoient à des expériences culturelles différentes. C'est ce qui transparaît dans l'exemple suivant (Conversation 2 ci-dessous), où l'alloglotte qui cherche à répondre à la question du natif bute sur une incompréhension lexicale. La valeur référentielle d'un mot que l'alloglotte connait, ou suppose connaitre, est différente de celle que le natif attribue à cette même forme. Ce fragment de conversation nous montre les efforts des deux interlocuteurs afin d'assurer l'intercompréhension par une reconstruction des valeurs référentielles et par la mise en relation des différentes valeurs, pour accéder au sens partagé.

Conversation 2: le dialogue se déroule entre un employé français $(\mathrm{F})$ et une standardiste thaie $(\mathrm{T})$, à l'Ambassade de France en Thailande ${ }^{6}$.

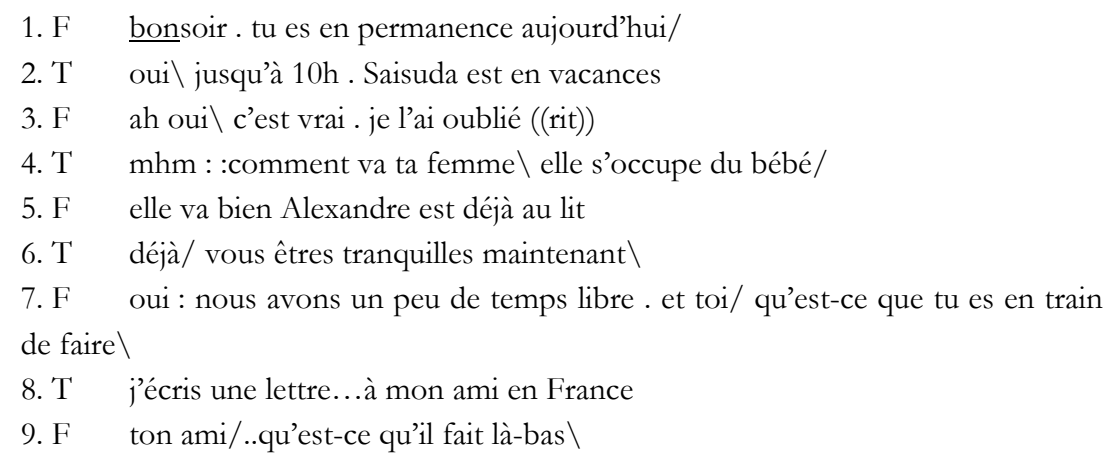

${ }^{6}$ Ce dialogue a été recueilli par T, thaillandaise, en septembre 1999. Après avoir obtenu une maittrise en français, elle a travaillé à l'ambassade de France à partir de 1991. Son interlocuteur français F y travaille comme gardien depuis 1996. Cette conversation a été recueillie à l'insu de F. 
Patchareerat Yanaprasart

10. T oh/ ça fait trois ans qu'il fait son doctorat à Paris

11. F Trois ans . ah ya ya/ Il te fait attendre depuis déjà trois ans/

12. $\mathrm{T}$ Comment ça

13. F je voulais dire . qu'il euh .. qu'il est parti depuis trois ans=et toi euh tu : : attends toujours son retour ici . c'est long . non/

14. $\mathrm{T}$ oui . mais . pourquoi attendre/ c'est un ami à moi . on a fait des études universitaires ensemble

15. F ah d'accord. il n'est pas ton petit ami

16. $\mathrm{T}$ mais non/ juste un ami proche

Dans cet exemple, ce sont des problèmes de type sémantico-référentiel qui sont à la source du malentendu autour de «mon ami» (8T). Certes, l'oral ne livre pas d'indice de genre (mon ami/mon amie). Mais à l'évidence, cela n'est pas source d'ambiguïté pour F (9F «ton ami qu'est-ce qu'il fait là-bas ?»), pour qui il s'agit du «petit ami», ce qui le pousse à manifester son étonnement avec une exclamation, «Trois ans! », pensant que sa collègue thaïe a passé autant de temps à attendre son petit ami. L'intonation montante de la phrase $(11 \mathrm{~F}$ « Il te fait attendre depuis déjà trois ans/ ») manifeste la surprise et/ou la taquinerie du locuteur. Interprétant cela comme une question, la standardiste se sent obligée d'y répondre. Elle réplique par une autre question : "Comment ça » (12T), équivalant à "je ne comprends pas ce que tu me demandes, ou pourquoi tu me le demandes". S'apercevant de la non-compréhension de sa colocutrice, le natif tente d'expliciter et d'argumenter $(13 \mathrm{~F}$ « je voulais dire que ...») sa question antérieure. Le fait que $\mathrm{F}$ substitue à sa première forme de question des éléments considérés comme plus accessibles à l'alloglotte résulte de sa volonté de clarifier la première formulation. Cependant, il semble que cela ne clarifie pas suffisamment pour T l'intention de son interlocuteur. C'est en 13-14 que se dénoue l'ambiguïté ( $13 \mathrm{~F}$ c'est long.non », 14T «pourquoi attendre »), reprenant le fil déjà amorcé $(10 \mathrm{~T}$ « trois ans », $11 \mathrm{~F}$ « trois ans $(\ldots)$ Il te fait attendre $(\ldots), 13 \mathrm{~F}$ «trois ans $(. .$.$) tu attends toujours (.) »$.$) . Le "pourquoi attendre» en 14 \mathrm{~T}$ ne fait que répercuter le «comment ça » de $12 \mathrm{~T}$ ("Pourquoi dis-tu 'attendre' et 'c'est long' ?"). Sans attendre la réponse de $\mathrm{F}$, puisqu'elle se rend tout à coup compte de la méprise, la standardiste remplace «mon ami » par « un ami à moi », de façon tout à fait conforme en cette occurrence à l'usage de la langue cible. Elle adopte une stratégie explicative en ajoutant "on a fait des études universitaires ensemble » afin de préciser la nature amicale de la relation. L'identification sémantique du lexème ami lève le malentendu. L'approbation de F exprimée par «ah d'accord, il n'est pas ton petit ami » entérine la rectification du sens visé par la Thailandaise, « juste un ami proche » (16T).

Nous voyons là une caractéristique essentielle du comportement conversationnel entre alloglotte et natif: le travail de coopération. La collaboration des interactants révèle leur effort de pallier le dysfonctionnement attaché à la divergence sémantique, par un travail discursif de rapprochement, et ce d'une manière interactive menant finalement à la compréhension mutuelle. Comme le postulent Alber et Py (1986, 83),

la réussite communicative en situation d'asymétrie linguistique dépend d'un travail accru d'intelligibilisation fondé sur la coopération des interlocuteurs, accompagné 
d'une répartition fonctionnelle des tâches entre le partenaire linguistiquement fort et le partenaire linguistique faible.

L'image dominante que cet exemple donne de l'alloglotte est celle d'un négociateur actif. Sa maittrise suffisante du français lui permettant d'identifier la cause du blocage conversationnel, elle demande des clarifications avec des interrogations explicites : "Comment ça », "pourquoi attendre», en tentant de construire des hypothèses interprétatives sur le sens visé par l'énoncé de son interlocuteur. Ces demandes de clarification lui paraissent nécessaires pour la poursuite de l'échange, car les requêtes réitérées du natif l'obligent à répondre de manière satisfaisante. La réaction d'incompréhension ou d'incertitude de l'alloglotte déclenche la reformulation ou l'explicitation de la question concernée. Sous sa forme paraphrastique, la reformulation, stratégie d'hétérofacilitation par excellence en conversation exolingue (Alber et Py 1986 : 87), a une fonction simplificatrice (simplifier le discours pour le rendre accessible), une fonction réparatrice (pallier les lacunes des connaissances en français, résoudre un problème de compréhension), et enfin une fonction constructive (établir l'intercompréhension), le but essentiel étant de permettre au dialogue de se poursuivre.

\section{Les divergences entre règles conversationnelles}

Quand deux systèmes de valeurs, de pratiques culturelles et d'actes rituels se rencontrent, des erreurs ou asymétries de perception réciproque se produisent. Comme on va le voir, les difficultés conversationnelles ne peuvent pas toutes être ramenées à des divergences d'ordre linguistique, même si celles-ci jouent un rôle considérable dans le déroulement des interactions. Les valeurs sous-jacentes que reflètent les pratiques discursives donnent en grande partie au message son sens global en contexte. Les relations interpersonnelles peuvent en être perturbées et sont souvent les plus affectées dans les contacts interculturels, quand la bonne impression que l'on veut donner de soi rencontre une incompréhension, une évaluation partielle, un jugement réciproque négatif, ou une représentation mutuelle faussée entraînant des réactions de rejet.

Conversation 3: la conversation se déroule au Lycée français de Bangkok, entre une secrétaire thaie (T) et un Belge (B), responsable de la vie scolaire ${ }^{7}$.
1. B sa : :lut Manida . ça va/
2. $\mathrm{T}$ oui je vais bien merci . et toi/
3. B bien . bien merci . euh . dis-moi . est-ce que : :t'as fini euh...la liste

\footnotetext{
${ }^{7} \mathrm{~T}$ a obtenu sa licence ès lettres en 1994. Mariée avec un Français, elle déménage à Bangkok. Embauchée au lycée en 1996, elle poursuit en même temps ses études de maitrise de traduction français-thaï. Séjournant depuis longtemps en Thailande, B parle très bien le thaï. Il ne parle que français avec la secrétaire. L'anglais est choisi lors de ses contacts avec d'autres Thailandais et le thai est réservé aux discussions avec les femmes de ménage, gardiens et techniciens. Ce dialogue a également été relevé d'une manière discrète par T.
} 
d'adresses des parents d'élèves/on=en a besoin cette après-midi . tu sais $\backslash$.. il faut qu'on prépare des billets de théâtre pour lundi prochain. on va les envoyer demain matin . peux-tu m'en dire le nombre total/

4. T en tout/c'est : : 400 familles

5. B ah ah : c'est moins que l'année passée \bon . tu demandes à Toun...de me l'apporter $\backslash$ Si possible . avant $10 \mathrm{~h} . .$. comme ça $=$ j'aurai le temps d'en parler avec le proviseur $\mathrm{mhm}:$ :pendant la récréation

6. T D'accord..je vais l'imprimer maintenant

7. B merci ((rit))

8. $\mathrm{T} \quad$ oui $\backslash$ (en souriant).

9. B mais/ il faut dire "de rien"..Dire "oui”" est impoli

Dans cette situation, une incompréhension est près de se produire lorsque la secrétaire thaïe, malgré sa compétence bilingue achevée, ne semble pas consciente de la différence de sens au niveau communicatif et pragmatique de la même expression verbale (oui) et non verbale (le sourire) dans les deux cultures. Sa réponse, en 8T, est un transfert pragma-linguistique calqué sur sa langue d'origine.

Le sourire, manière non verbale d'exprimer l'acceptation du remerciement selon la règle thaïe, n'est pas une condition de réussite de l'acte des «chaînes » de remerciement dans la culture du locuteur francophone. Bien que la réplique « oui » soit verbalisée, elle n'a pas la même valeur illocutoire dans les deux langues, ce qui peut avoir pour conséquence un malentendu entre les interactants. Puisque la conversation se déroule en français, le discours est censé s'organiser selon les règles pragma-linguistiques et socio-pragmatiques françaises. Cela explique pourquoi le locuteur natif adopte une attitude pédagogique en corrigeant son interlocutrice, fût-ce pour le principe et sur un mode conniventiel (9. B « il faut dire 'de rien'..Dire 'oui' est impoli »).

Cette indication ou injonction de B $(9 \mathrm{~B})$ de corriger « oui » par « de rien » ne provient pas de son ignorance de l'interférence culturelle entre la langue source et la langue cible, mais peut-être d'une interrogation sur les conventions discursives utilisées par la Thailandaise: cette lacune provient-elle de son refus d'adopter les normes conversationnelles françaises ou d'une méconnaissance d'usages sociolinguistiques? En tous cas, cette forme d'hétérocorrection (Py 1995), effectuée spontanément, même si elle a pour but d'instruire la compétence de l'alloglotte, souligne en même temps une infériorité de celle-ci. Une telle attitude pédagogique, traduite par la correction explicite ou professorale (Jefferson 1983) impliquant une relation du type maître-élève, sans contrat didactique légitimé (De Pietro, Matthey, Py 1989) ou «contrat de parole» (Charaudeau 1983), menace le partenaire faible de perdre la face (Goffman 1974).

Encore une fois, le malentendu interculturel porte plus souvent sur le contenu implicite que sur le contenu littéral du message. C’est au niveau méta (quel est le sens de ce mot dans ce contexte? Que veut me dire la personne avec ce mot-là? Qu'attend-elle de moi? Comment dois-je lui répondre?) que se trouvent les difficultés. Cette interaction nous amène à une conclusion : il faut prendre en compte le fait que les malentendus ne sont pas toujours réductibles à des divergences 
linguistiques. Une bonne partie des malentendus interculturels proviennent aussi d'éléments extralinguistiques ainsi que de différences dans les règles conversationnelles. Les participants interprètent paroles et actions à travers la grille de lecture de leur culture d'origine, et leur attribuent le sens qu'elles auraient dans leur propre culture.

Dans le cas suivant (Conversation 4), on observe un conflit portant sur les valeurs et la relation interpersonnelle. Ce type de conflit est lié à la façon dont les interactants évaluent la situation, le rôle que chacun a à jouer et le type de relation qu'ils établissent dans la situation. L'interaction devient problématique lorsque les interactants sont en désaccord sur la façon de traiter les autres ou d'être traités par eux.

Conversation 4 : l'interaction a lieu entre un élève péruvien $(\mathrm{P})$ et sa camarade thaïe $(\mathrm{T})$ à Neuchâtel ${ }^{8}$.
1. P salut. ça va/
2. $\mathrm{T}$ be...bien . et toi $\backslash$
3. P ça va. on se fait la bise/ [il rit]
4. $\mathrm{T}$ no: :n..euh pas de bise
5. P mais : : pourquoi/ on se connait bien eh..non/
6. T ce n'est pas la question . c'est . j- je suis Thailandaise et toi=tu n'es pas Suisse \ce n'est pas ma façon de .. de saluer pour : : :pourquoi on doit faire comme les autres $\backslash$ chez toi... on fait comment $\backslash$
7. P ouh :/quelle importance/ on est en $\underline{\text { Suisse }}$
8. T $\mathrm{mhm}:: \backslash$ nous ne sommes pas...Suisses

Dans cet exemple, l'interaction est rendue conflictuelle par la façon dont les interactants interprètent le sens global implicite du geste en termes de relations interpersonnelles. La matière même de cette interaction concerne les valeurs, les attitudes, les croyances et les comportements qu'on prête à la façon de saluer. Même si les participants partagent l'idée que la bise est autorisée entre gens se connaissant bien, l'un des deux (T) refuse cette pratique gestuelle, qui n'est ni la sienne ni celle de son interlocuteur. Cela explique pourquoi la proposition exprimée par le Péruvien : « on se fait la bise/» (3P) n'est pas acceptée par sa camarade. La raison invoquée par le premier (« on se connait bien », 5P) ne semble pas assez convaincante pour que la seconde accomplisse cet acte de salutation, puisqu'elle perçoit cette façon de saluer comme une forme employée entre Suisses. En premier lieu, elle fait allusion à son identité culturelle : «je suis Thailandaise (...) ce n'est pas ma façon de saluer» (6T). Puis, elle identifie son interlocuteur par rapport aux Suisses et lui explique que lui aussi est étranger : « tu n'es pas Suisse (...) chez toi, on fait comment ? (6T). Ensuite, la réplique ("nous ne sommes pas Suisses », 8T) à la séquence argumentative de $\mathrm{P}$ (« on est en Suisse »,7P) implique pour la Thailandaise qu'il n'est pas nécessaire de se

\footnotetext{
${ }^{8}$ Cette interaction a été enregistrée pendant une récréation durant l'année scolaire 1999-2000. T est une jeune thailandaise venue en Suisse après le mariage de sa mère avec un Suisse six ans plus tôt. Elle est en dernière année à l'École de commerce. Le Péruvien a vécu à Neuchâtel pendant dix ans. Il se prépare à quitter la Suisse après la fin de ses études en 1999.
} 
conformer aux normes interactionnelles locales puisque que ni lui ni elle ne sont autochtones. Cela laisse entendre, nous semble-t-il, que si l'un des participants était Suisse, elle pourrait se permettre d'adopter cette norme comportementale. L'essentiel du problème semble tenir en partie à la relation homme-femme, à l'expression corporelle, à la manifestation d'un contact physique en public. Est-il possible que le Péruvien tente de profiter de cette pratique en tant que garçon et que cette Thailandaise " traditionnaliste" ne l'accepte pas par pudeur ? D'autre part, les conflits d'identités apportent un autre éclarage à cet épisode inter-culturel, quant aux différences d'attitude par rapport à la culture d'origine et à la culture de l'univers d'accueil: l'un des deux étrangers (P) éprouve la volonté de se conduire selon les pratiques sociales du pays de séjour, l'autre ne fait pas le même effort pour s'y habituer ou s'y conformer. Cette dernière $(T)$ construit ou préserve son identité culturelle en demandant: "pourquoi on doit faire comme les autres? ? (6T), pour mettre en évidence l'altérité culturelle. Pour elle, la bise est un comportement ritualisé qui fonctionne comme un marqueur identitaire. Refuser ce marqueur d'identité suisse est sa façon de nier son appartenance à la communauté et d'affirmer son identité étrangère. Pour l'autre (ici le Péruvien), s'il se trouve dans un pays où la bise constitue une convention culturelle d'interaction, il lui parait raisonnable de se soumettre aux normes qui y sont pratiquées. Cet exemple nous montre le travail de construction identitaire. Par leurs comportements et leurs propos, les interlocuteurs se donnent mutuellement une image et une définition d'eux-mêmes (Ladmiral et Lipiansky 1989).

Cette séquence d'interaction, intéressante au plan socioculturel, comporte également des traces linguistiques, d'autant plus qu'il s'agit d'une interaction en français entre locuteurs non natifs. Le segment problématique «pas de bise» (4T) de la Thailandaise a amené le Péruvien à le «décontextualiser» (Py 1994b), c'est-à-dire à l'extraire du contexte d'occurrence. Pour ce faire, ce dernier a recours à une interrogation («pourquoi », 5P) dont la force illocutoire de demande d'explication ou de justification est ainsi linguistiquement marquée . L'énoncé interrogatif «pourquoi » vise, de plus, à «ménager la face négative» du locuteur (Brown et Levinson, 1987) dans le sens où ce qu'il avait proposé était présumé conforme à la norme pragmatique et sociale d'un locuteur natif de la langue de l'échange dans le pays, la région ou la ville du contexte. Cette stratégie dite d'«auto-justification» consiste à assurer de l'intercompréhension et à écarter le malentendu dû à la différence d'interprétation de la réalité sociolinguistique.

\section{Conclusion}

Comme on l'a postulé, le malentendu interculturel provient de la rencontre de deux systèmes différents, d'où les divergences d'interprétation. Les difficultés de communication rencontrées par nos interlocuteurs sont soit des problèmes de compétence linguistique, soit des problèmes aux niveaux pragma-linguistique et sociopragmatique. Dans le premier cas, on observe des malentendus portant sur le sens littéral des énoncés; dans le deuxième cas, sur le sens global implicite. En raison de l'asymétrie linguistique et culturelle, la communication entre interlocuteurs de cultures différentes exige autant d'efforts coopératifs linguistiques qu'extralinguistiques pour 
assurer la construction commune de l'interaction. Cette présentation a mis en lumière des stratégies discursives développées spontanément par les interlocuteurs pour élaborer le sens commun du message, faciliter le bon déroulement de la communication et faire circuler l'information en dépit des obstacles à l'intercompréhension.

Dans cet article, nous avons observé l'apparition d'obstacles à la communication ainsi que certains procédés linguistiques de nature à pallier les difficultés provoquées par les divergences de codes initiales (appels à l'aide, offres de collaboration, etc.), mis en œuvre par les interlocuteurs pour assurer un déroulement coopératif de la conversation. Cette analyse conversationnelle des interactions nous permet non seulement de mieux comprendre comment un alloglotte réagit lorsqu'il se trouve en conversation avec un natif de la langue cible ou à quelles stratégies de communication il recourt pour surmonter les difficultés et résoudre les malentendus, mais aussi de percevoir de quelle manière le natif collabore avec son partenaire pour combler les lacunes ou simplifie un système qu'il maitrise afin d'en faciliter la compréhension à son interlocuteur. Nos exemples témoignent d'un effort de construction de la compréhension mutuelle et d'une coopération pour la réussite de la conversation. L'étude d'interactions exolingues éclaire le processus d'établissement de l'intercompréhension à travers l'enchaînement de paroles et l'interaction. La synchronisation interactionnelle nécessite un travail collaboratif. A partir d'un corpus de situations communicatives asymétriques, il apparaît que les échanges se font souvent sur un mode conflictuel, qui peut prendre de nombreuses formes. Nous pouvons aussi voir des liens entre les structures conversationnelles et les relations de place (attitude pédagogique, rôle d'enseignant et d'évaluateur), ainsi que divers procédés d'ajustement - sollicitation, réparation, négociation, clarification, explication, simplification, facilitation, reformulation, auto-hétéro-correction, décontextualisation-recontextualisation (Alber et Py 1986, Berthoud 1986, De Pietro 1988, Py 1994a et 1995) — que les partenaires mettent en œuvre.

patchareerat.yanaprasart@unine.ch 


\section{BIBLIOGRAPHIE}

ALBER, J.-L. \& PY, B. (1985) : «Interlangue et conversation exolingue », Cabiers du département des langues et des sciences du langage 1, Lausanne, Université de Lausanne, 30-47.

ALBER, J.-L. \& PY, B. (1986): «Vers un modèle exolingue de la communication interculturelle : interparole, coopération et conversation », Etudes de linguistique appliquée 61, 78-90.

BAKHTINE, M. (1929) : Le marxisme et la philosophie du langage. Essai d'application de la méthode sociologique en linguistique. Paris, Les Editions de Minuit [trad. française de M. Yagüello, 1977].

BANGE, P. (1992) : Analyse conversationnelle et théorie de l'action. Paris, Hatier-Didier.

BERTHOUD, A.-C. (1986) : « Ambiguïté, malentendu et activité paradiscursive », Tranel 11.

BOUCHARD, R., DE NUCHEZE, V. (1987) : «Formulations métalangagières et situations exolingues », dans BLANC, H., LE DOUARON, M. \& VERONIQUE, D. (dir.), S'approprier une langue étrangère. Paris, Didier Érudition, 55-62.

BROWN, P. \& LEVINSON, S.-C. (1987): Politeness. Some Universals in Language Usage. Cambridge, Cambridge University Press, Studies in Interactional Sociolinguistics 4.

CALBRIS, G. et MONTREDON, J. (1986) : Des gestes et des mots pour le dire. Paris, CLE international.

CHARAUDEAU, P. (1983) : Langage et discours. Eléments de sémiolinguistique (Théorie et pratique). Paris, Hachette.

DE HEREDIA, C. (1986) : «Intercompréhension et malentendus. Etude d’interactions entre étrangers et autochtones ", Langue française 71, 48-69.

DE HEREDIA, C. (1987) : "Tuteurs et cache-pots ou le maitre quincailler : étude sur les malentendus en situation exolingue », dans H. BLANC, M. LE DOUARON ET D. VERONIQUE (dirs.) S'approprier une langue étrangère (Actes du Vie colloque international "Acquisition d'une langue étrangère: perspectives et recherches»). Paris, Didier Erudition, 23-31.

DE HEREDIA-DEPREZ, C. (1990) : «Intercompréhension et malentendus. Etude d'interactions entre étrangers et autochtones », dans F. FRANÇOIS (dir.) La communication inégale. Heurs et malbeurs de l'interaction verbale, Neuchâtel-Paris, Delachaux et Niestlé, 213-238.

DE PIETRO, J. F. (1988): «Conversations exolingues, une approche linguistique des interactions interculturelles ", dans COSNIER J., GELAS N., KERBRATORECCHIONI, C. (dirs.), Echanges sur la conversation. Paris, Editions du CNRS.

DE PIETRO, J. F., MATTHEY, M., PY, B. (1989) : «Acquisition et contrat didactique : les séquences potentiellement acquisitionnelles de la conversation exolingue », dans D. WEIL, H. FUGIER (éd.), Actes du troisième colloque régional de linguistique, Strasbourg, Université des sciences humaines et Université Louis Pasteur. 
FLAHAULT, F. (1978), La parole intermédiaire, Paris, Editions du Seuil.

GASS, S. M. et VARONIS, E. M. (1991), "Miscommunication in Non-Native Speaker Discourse", in COUPLAND et al., Miscommunication and Problematic Talk, London, Sage Publications.

GIACOMI, A., HOUDAÏFA, E.T. \& VION, R. (1984), « Malentendus et/ou incompréhensions dans la communication interculturelle: A bon entendeur, salut!», dans C. NOYAU \& R. PORQUIER (dirs.) : Communiquer dans la langue de l'autre. Paris, Presses universitaires de Vincennes 79-98.

GOFFMAN, E. (1959): The presentation of Self in Everyday Life. New York, Doubleday \& Company [trad. française d'A. Accardo: La mise en scène de la vie quotidienne 1, «La présentation de soi », Paris, Les Editions de Minuit, 1973].

GOFFMAN, E. (1974) : Les rites d'interaction. Paris, Editions de Minuit.

GUMPERZ, J. (1982) : Discourse Strategies. Cambridge University Press.

HAMMERLY, H. (1982) : Synthesis in Second Language Teaching : An Introduction to Linguistics. New York, Simon Fraser University Press.

HOLMES, H. (1996) : Working with the Thais. Bangkok, White Lotus.

HYMES, D.-H. (1991) : Vers la compétence de communication. Paris, Crédif-Hatier/Didier, coll. LAL [Trad. fr. de F. Mugler de textes de 1973 et 1982].

JEFFERSON, G. (1983) : “On Exposed and Embedded Correction in Conversation”, Studium linguistik 14, Hain, Dieter Wunderlich, 58-68.

KERBRAT-ORECCHIONI, C. (1990) : Les interactions verbales, tome I. Paris, Armand Colin.

LADMIRAL, J.-R. et LIPIANSKY, E. M. (1989) : La communication interculturelle. Paris, Armand Colin.

LINTON, R. (1945) : The cultural Background of Personality. New York, Appleton-Century-Crofts [trad. fr. d'A. Lyotard : Le fondement culturel de la personnalité, Paris, Monographies Dunod, 1967].

OERTIG-DAVIDSON, M. (2002) : Beyond Chocolate. Basel, Bergli Books.

PORQUIER, R. (1984) : «Communication exolingue et apprentissage des langues », dans B. PY (dir.) Encrages, Actes du colloque « Acquisition d'une langue étrangère III » organisé à l'université de Neuchâtel les 16-18 septembre 1982, Paris, Presses de l'Université de Paris VIII-Vincennes, 17-47.

PORQUIER, R. (1994) : «Communication exolingue et contextes d'appropriation: Le continuum acquisition / apprentissage », dans B. PY (dir.) : L'acquisition d'une langue seconde, Bulletin suisse de linguistique appliquée, 59, 159-170.

PY, B. (1994): «Linguistique de l'acquisition des langues étrangères: naissance et développement d'une problématique », dans D. COSTE (dir.) : Vingt ans dans l'évolution de la didactique des langues (1968-1988). Paris, Didier. 
PY, B. (1994b) : « Place des approches interactionnistes dans l'étude des situations de contacts et d'acquisition ", dans D. VERONIQUE (dir.), Créolisation et acquisition des langues. Aixen-Provence, Publications de l'Université de Provence, 137-150.

PY, B. (1995) : «Interaction exolingue et processus d'acquisition », Cabiers de l'ILSL 7.

SELINKER, L. (1972) : «Interlanguage », International Review of Applied Linguistics 10-1, 209-231.

SPERBER, D. et WILSON, D. (1986/1989) : La pertinence. Paris, Minuit.

TRÉVISE, A. et DE HEREDIA, C. (1984) : «Les malentendus : effets de loupe sur certains phénomènes d'acquisition d'une langue étrangère", dans C. NOYAU \& R. PORQUIER (dirs.) : Communiquer dans la langue de l'autre. Paris, Presses Universitaires de Vincennes, 130-152.

VION, R. (1992) : La communication verbale. Analyse des interactions. Paris, Hachette.

WATZLAWICK, P., BEAVIN, J. H., JACKSON D. (1972) : Une logique de la communication. Paris, Seuil [trad. de Pragmatics of Human Communication, 1967].

YANAPRASART, P. (2000) : Langue et culture dans l'enseignement du français en Thaïlande. Thèse de doctorat en linguistique appliquée, Université de Neuchâtel. 